\title{
ИДЕНТИФИКАЦИЯ ТРЕНДОВ И ВОЛНОВЫХ ЗАКОНОМЕРНОСТЕЙ ПО СТАТИСТИЧЕСКИМ ДАННЫМ КОЛЕБАНИЙ МАССЫ ЛЕДНИКОВ
}

\author{
Мазуркин П.М. \\ Д-р тех. наук, проф., академик ЕАЕ, РАЕ и РАЕН, \\ Поволжский государственный технологический университет, Йошкар-Ола, kaf_po@mail.ru
}

Анотация Метод идентификации показан на примере табличных данных измерений шести параметров подгруппы ЕЕЕ по колебаниям в 2013 году у баланса массы 2528 ледников Земли. Получены уравнения тренда и колебательных возмущений на основе устойчивых законов по обобщенной волновой функции в виде асимметричного вейвлет-сигнала с переменными амплитудой и периодом колебания. Графики составляющих обобщенной модели вейвлет-сигнала позволяют наглядно увидеть картину взаимного влияния всех шести параметров подгруппы ЕЕЕ у колебаний массы ледников. По выявленным уравнениям можно провести амплитудночастотный анализ.

Ключевые слова: ледники, колебание, баланс массы, факторы, закономерности

\section{1. Введение}

Мы придерживаемся концепции Декарта о необходимости применении алгебраического уравнения общего вида напрямую как конечного математического решения неизвестных дифференциальных или интегральных уравнений. Для такого обобщения был предложен новый класс волновых функций [1-8, 10-12].

Условиям физического существования явлений и процессов наиболее полно удовлетворяет асимметричная вейвлет-функция вида

$$
y=\sum_{i=1}^{m} y_{i}, y_{i}=a_{1 i} x^{a_{2 i}} \exp \left(-a_{3 i} x^{a_{4 i}}\right) \cos \left(\pi x /\left(a_{5 i}+a_{6 i} x^{a_{7 i}} \exp \left(-a_{8 i} x^{a_{9 i}}\right)-a_{10 i}\right),\right.
$$

где $y$ - показатель (зависимый фактор), $i$ - номер составляющей модели (17), $m$ - количество членов в модели (1), $x$ - объясняющая переменная (влияющий фактор), $a_{1} \ldots a_{10}$ - параметры, принимающие числовые значения в ходе структурно-параметрической идентификации (URL: http://www.curveexpert.net/) по мере увеличения коэффициента корреляции при наращивании количества составляющих статистической модели (1).

Сигнал - это материальный носитель информации. А информация нами понимается как мера взаимодействия. Сигнал может генерироваться, но его приём не обязателен. Так, например, ряд простых чисел известен несколько тысяч лет, но суть его как множества сигналов до сих пор не была раскрыта [12].

Сигналом может быть любой физический процесс или его часть. Получается, что изменение множества неизвестных сигналов давно известно, например, через ряды гидрометеорологических измерений во многих точках планеты. Однако до сих пор нет статистических моделей динамики глобальной температуры и других параметров земной среды.

В большинстве случаев для идентификации искомых закономерностей по известным табличным моделям достаточна усеченная конструкция (по формуле частоты колебания) асимметричного вейвлета [10-12] типа

$$
y=\sum_{i=1}^{m} y_{i}, y_{i}=a_{1 i} x^{a_{2 i}} \exp \left(-a_{3 i} x^{a_{4 i}}\right) \cos \left(\pi x /\left(a_{5 i}+a_{6 i} x^{a_{7 i}}\right)-a_{8 i}\right),
$$

где $y$ - показатель (зависимый фактор), $y_{i}=A_{i} \cos \left(\pi x / p_{i}-a_{8 i}\right), i$ - номер составляющей модели (2), $m$ - количество членов в модели (2), $x$ - объясняющая переменная (влияющий фактор), $a_{1} \ldots a_{8}$ - параметры, принимающие числовые значения в ходе структурно-параметрической идентификации формулы (2), $A_{i}=a_{1 i} x^{a_{2 i}} \exp \left(-a_{3 i} x^{a_{4 i}}\right)$ - амплитуда (половина) асимметричного 
вейвлета (ось $y$ ), $p_{i}=a_{5 i}+a_{6 i} x^{a_{7 i}}$ - полупериод волны колебательного возмущения (ось $x$ ).

По формуле (2) с двумя фундаментальными физическими постоянными $е$ (число Непера или число времени) и $\pi$ (число Архимеда или число пространства) образуется изнутри изучаемого явления и/или процесса квантованный вейвлет-сигнал.

Понятие вейвлет-сигнала позволяет абстрагироваться от физического смысла статистических рядов измерений (в общем случае не только динамических рядов, но и любых других бинарных отношений между факторами) и рассматривать их аддитивное разложение на отдельные составляющие в виде вейвлетов.

\section{2. Исходные данные для статистического моделирования}

В геоэкологии нами рассматриваются разные виды природных объектов [6], и среди них, несомненно, важное место занимают ледники, как регуляторы климата. В статье закономерности распределения ледников за 2013 год даны впервые.

По исходным данным [9] в таблице 1 показан фрагмент матрицы, включающей шесть факторов (идентификаторы приняты по оригиналу).

Таблица 1. Матрица исходных данных параметров ледников для статистического моделирования

\begin{tabular}{|c|c|c|c|c|c|c|}
\hline $\begin{array}{c}\text { № } \\
\Pi / \Pi\end{array}$ & $\begin{array}{l}\text { Широта } \\
\text { ЕЕЕ6, } \\
\text { град } \\
\end{array}$ & $\begin{array}{c}\text { Долгота } \\
\text { ЕEЕ7, } \\
\text { град } \\
\end{array}$ & $\begin{array}{c}\text { Высота EEE8 } \\
\text { над уровнем } \\
\text { моря, м } \\
\end{array}$ & $\begin{array}{c}\text { Зимний баланс } \\
\text { массы } \\
\text { EEE9, мм }\end{array}$ & $\begin{array}{c}\text { Летний баланс } \\
\text { массы } \\
\text { EEE10, мм } \\
\end{array}$ & $\begin{array}{c}\text { Годовой баланс } \\
\text { массы } \\
\text { EEE11, мм } \\
\end{array}$ \\
\hline 1 & & & 100 & & & 100 \\
\hline 2 & & & 398 & & & 500 \\
\hline$\ldots$ & $\ldots$ & $\ldots$ & $\ldots$ & $\ldots$ & $\ldots$ & $\ldots$ \\
\hline 71 & & & 1073 & & & -63 \\
\hline 72 & -54.78136 & -68.40169 & 1038 & 1015 & -644 & 371 \\
\hline 73 & -54.78182 & -68.40249 & 1036 & 947 & -540 & 406 \\
\hline$\ldots$ & $\ldots$ & $\ldots$ & $\ldots$ & $\ldots$ & $\ldots$ & $\ldots$ \\
\hline 101 & -54.78048 & -68.40171 & 1073 & 743 & -984 & -241 \\
\hline 102 & -16.3032 & -68.108 & 5053 & & & -958 \\
\hline 103 & -16.3025 & -68.1083 & 5056 & & & -1385 \\
\hline$\ldots$ & $\ldots$ & $\ldots$ & $\ldots$ & $\ldots$ & $\ldots$ & $\ldots$ \\
\hline 620 & & & 4058 & & & 120 \\
\hline 621 & & & 3796 & 20 & -3719 & -3699 \\
\hline 622 & & & 3828 & 99 & -2565 & -2466 \\
\hline$\ldots$ & $\ldots$ & $\ldots$ & $\ldots$ & $\ldots$ & $\ldots$ & $\ldots$ \\
\hline 2526 & 60.40417 & -148.9067 & 1053 & & & -2090 \\
\hline 2527 & 60.41974 & -148.9207 & 1283 & & & 0 \\
\hline 2528 & 60.42495 & -148.9371 & 1367 & & & 960 \\
\hline
\end{tabular}

Данные расположены несимметрично (табл. 2).

Таблица 2. Количество измеренных точек баланса массы ледников, шт.

\begin{tabular}{|l|c|c|c|c|c|c|}
\hline \multirow{2}{*}{$\begin{array}{c}\text { Influencing } \\
\text { factors } x\end{array}$} & \multicolumn{5}{c|}{ Dependent factors (indicators $y$ ) } \\
\cline { 2 - 7 } & EEE6 & EEE7 & EEE8 & EEE9 & EEE10 & EEE11 \\
\hline EEE6 - POINT LATITUDE [decimal degree] & 1623 & 1623 & 1623 & 388 & 388 & 1591 \\
\hline EEE7 - POINT LONGITUDE [decimal degree] & 1623 & 1623 & 1623 & 388 & 388 & 1591 \\
\hline EEE8 - POINT ELEVATION [m a.s.l.] & 1623 & 1623 & 2467 & 531 & 531 & 2467 \\
\hline EEE9 - POINT WINTER BALANCE [mm w.e.] & 388 & 388 & 531 & 531 & 531 & 531 \\
\hline EEE10 - POINT SUMMER BALANCE [mm w.e.] & 388 & 388 & 531 & 531 & 531 & 531 \\
\hline EEE11 - POINT ANNUAL BALANCE [mm w.e.] & 1591 & 1591 & 2467 & 531 & 531 & 2467 \\
\hline
\end{tabular}

Каждый из факторов может иметь два состояния: во-первых, фактор как влияющая переменная $x$; во-вторых, этот же фактор принимается как зависимый показатель $y$. По ним можно 
допустить, что амплитуда и период колебаний по общей модели (2) подчиняются биотехническому закону [1-8]. Из-за неопределенности направления вектора «лучше $\rightarrow$ хуже» у значений каждого фактора не выявляем закономерности ранговых распределений. Поэтому рейтинг ледников также не проводим.

В связи с этим коэффициент корреляции ранговых распределений равен 1 , а факторный анализ проведем у всех $6^{2}-6=30$ бинарных отношений.

\section{3. Рейтинг факторов как влияющих переменных и показателей}

Предлагаемый нами метод факторного анализа позволяет не задумываться априори о соотношениях между отдельными параметрами изучаемой системы (в нашем примере из таблицы 1 система содержит 2528 ледников). В итоге сбивается психологический барьер у исследователей: многие бинарные отношения для них окажутся неожиданными. Поэтому, как показала наша практика [1], факторный анализ одним единственным уравнением типа (1) позволяет находить неожиданные научные решения в области исследования [1-8]. Если некоторые факторные связи необычны и при этом высоко адекватны, то здесь теоретически проявляются новые технические решения. Причем зачастую на уровне изобретений мировой новизны [11]. При этом повторяющийся процесс идентификацию закона (1) на одном бинарном отношении мы назвали вейвлет-анализом [2, 5].

Без учета волн, то есть изменения только по амплитуде у очень длинной волны, несоизмеримо большей по периоду колебания интервалу измерений, образуются так называемые детерминированные бинарные отношения. Они все являются частными случаями формулы (2).

В таблице 3 приведена квадратная корреляционная матрица, полученная после анализа бинарных отношений между всеми принятыми по исходным данным из таблицы 1 шестью переменными величинами. Здесь же дан рейтинг факторов.

Таблица 3. Корреляционная матрица и рейтинг факторов по бинарным отношениям

\begin{tabular}{|c|c|c|c|c|c|c|c|c|}
\hline \multirow{2}{*}{$\begin{array}{c}\text { Influencing } \\
\text { factors } x\end{array}$} & \multicolumn{6}{|c|}{ Dependent factors (indicators $y)$} & Sum & Place \\
\cline { 2 - 8 } & EEE6 & EEE7 & EEE8 & EEE9 & EEE10 & EEE11 & $\sum r$ & $I_{x}$ \\
\hline EEE6 & 1 & $\mathbf{0 . 9 7 3 2}$ & 0.9491 & 0.4425 & 0.3985 & $\mathbf{0 . 4 5 5 1}$ & 4.2184 & 2 \\
\hline EEE7 & $\mathbf{0 . 9 6 8 8}$ & 1 & 0.4305 & 0.4154 & 0.4629 & 0.4156 & 3.6932 & 3 \\
\hline EEE8 & $\mathbf{0 . 8 1 7 8}$ & $\mathbf{0 . 8 6 1 1}$ & 1 & $\mathbf{0 . 7 2 0 1}$ & $\mathbf{0 . 6 8 3 5}$ & $\mathbf{0 . 4 4 2 1}$ & 4.5246 & $\mathbf{1}$ \\
\hline EEE9 & 0.2237 & 0.4492 & 0.5431 & 1 & 0.2265 & 0.4721 & 2.9146 & 6 \\
\hline EEE10 & 0.6408 & 0.5571 & 0.1557 & 0.2301 & 1 & 0.7309 & 3.3146 & 5 \\
\hline EEE11 & 0.3703 & 0.4978 & 0.1719 & 0.5363 & 0.7592 & 1 & 3.3355 & 4 \\
\hline Sum $\sum r$ & 4.0214 & 4.3384 & 3.2503 & 3.3444 & 3.5306 & 3.5158 & 22.0009 & - \\
\hline Place $I_{y}$ & 2 & $\mathbf{1}$ & 6 & 5 & 3 & 4 & - & 0.6111 \\
\hline
\end{tabular}

Примечание. Выделены тренды с волновой составляющей.

Коэффициент коррелятивной вариации для 2528 ледников Земли равен $22.0009 / 6^{2}=$ 0.6111. Этот критерий применяется при сравнении различных систем, например, разных групп ледников друг с другом. Возможно также сравнение всех ледников с другими объектами нашей планеты, например, с пустынями или с агроэкологическими классами почвенного покрова.

Из шести влияющих переменных на первом месте оказался фактор EEE8. На втором месте поместился фактор EEE6, а на третьем месте - EЕE7. Среди зависимых показателей на первом месте оказывается фактор EEE7. На втором месте расположился фактор EEE6, а на третьем EEE10.

\section{4. Сильные бинарные отношения}

При коэффициенте корреляции более 0.7 бинарные отношения между факторами становятся сильными (табл. 4). Как правило, дополнительный к тренду учет волнового возмущения дает значительный рост адекватности выявляемой закономерности по формуле (2). Но ледники пока не имеют добротных данных, так как многие значения у шести факторов имеют пустые 
клетки: наилучшей матрицей является таблица с полностью заполненными клетками.

Из-за низкой полноты матрицы из 30 формул получили всего восемь сильных связей, что составляет 22.2\%. При этом как влияющая переменная фактор EEE9 - POINT WINTER BALANCE исключился. Но как зависимые показатели сохранились все шесть факторов. Из восьми сильных связей три (37.50\%) относятся к трендам.

Таблица 4. Корреляционная матрица сильных бинарных отношений при $r>0.7$

\begin{tabular}{|l|c|c|c|c|c|c|}
\hline \multirow{2}{*}{ Влияющие факторы } & \multicolumn{5}{|c|}{ Зависимые факторы (показатели $y$ ) } \\
\cline { 2 - 7 } & EEE6 & EEE7 & EEE8 & EEE9 & EEE10 & EEE11 \\
\hline EEE6 - POINT LATITUDE & & $\mathbf{0 . 9 7 3 2}$ & 0.9491 & & & \\
\hline EEE7 - POINT LONGITUDE & $\mathbf{0 . 9 6 8 8}$ & & & & & \\
\hline EEE8 - POINT ELEVATION & $\mathbf{0 . 8 1 7 8}$ & $\mathbf{0 . 8 6 1 1}$ & & $\mathbf{0 . 7 2 0 1}$ & & \\
\hline EEE10 - POINT SUMMER BALANCE & & & & & & 0.7309 \\
\hline EEE11 - POINT ANNUAL BALANCE & & & & & 0.7592 & \\
\hline
\end{tabular}

Иерархия сильных связей следующая (табл. 5): 1) 0.9732 - EEE7 = f(EEE6); 2) 0.9688 EEE6 = f(EEE7); 3) $0.9491-$ EEE8 = f(EEE6); 4) $0.8611-$ EEE7 = f(EEE8); 5) $0.8178-$ EEE6 = $\mathrm{f}($ EEE8); 6) $0.7592-$ EEE10 = f(EEE11); 7) $0.7309-$ EEE11 = f(EEE10); 8) $0.7201-$ EEE9 = $\mathrm{f}(\mathrm{EEE} 8)$.

В таблице 5 (рис. 1-7) даны параметры статистических моделей по общей формуле (2), значения которых записаны в компактной матричной форме с пятью значащими цифрами.

Таблица 5. Параметры сильных бинарных отношений при коэффициенте корреляции $r \geq 0,7$

\begin{tabular}{|c|c|c|c|c|c|c|c|c|c|}
\hline \multirow{3}{*}{$\begin{array}{c}\text { Показатели } \\
y\end{array}$} & \multicolumn{8}{|c|}{ Асимметричный вейвлет $y_{i}=a_{1 i} x^{a_{2 i}} \exp \left(-a_{3 i} x^{a_{4 i}}\right) \cos \left(\pi x /\left(a_{5 i}+a_{6 i} x^{a_{7 i}}\right)-a_{8 i}\right)$} & \multirow{3}{*}{$\begin{array}{c}\text { Коэф. } \\
\text { коррел. } \\
r \\
\end{array}$} \\
\hline & \multicolumn{4}{|c|}{ Амплитуда (половина) колебания } & \multicolumn{3}{|c|}{ Полупериод колебания } & \multirow{2}{*}{\begin{tabular}{|c} 
СдВиг \\
$a_{8 i}$ \\
\end{tabular}} & \\
\hline & $a_{1 i}$ & $a_{2 i}$ & $a_{3 i}$ & $a_{4 i}$ & $a_{5 i}$ & $a_{6 i}$ & $a_{7 i}$ & & \\
\hline \multicolumn{10}{|c|}{ Влияние широты EEE6, град } \\
\hline \multirow{3}{*}{$\begin{array}{l}\text { EEЕ7 }{ }^{1} \\
\text { Рис. } 1\end{array}$} & 4.23078 & 0 & -0.16452 & 0.54045 & 0 & 0 & 0 & 0 & \multirow{3}{*}{0.9732} \\
\hline & -0.31020 & 1.48076 & 0 & 0 & 0 & 0 & 0 & 0 & \\
\hline & 132.72384 & 0 & $8.79872 \mathrm{e}-5$ & 1.76602 & 4.87479 & 0.29176 & 0.56033 & 0.24849 & \\
\hline \multirow{2}{*}{$\begin{array}{l}\text { EЕE8 } \\
\text { Рис. } 1\end{array}$} & $1.66509 \mathrm{e} 6$ & 0 & 0.31234 & 0.94742 & 0 & 0 & 0 & 0 & \multirow{2}{*}{0.9491} \\
\hline & $7.82146 \mathrm{e}-5$ & 4.91282 & 0.018337 & 1.20027 & 0 & 0 & 0 & 0 & \\
\hline \multicolumn{10}{|c|}{ Влияние долготы EEE7, градус } \\
\hline \multirow{4}{*}{$\begin{array}{l}\text { EEE6 }{ }^{2} \\
\text { Рис. } 2\end{array}$} & 64.56655 & 0 & 0.00076040 & 1.01838 & 0 & 0 & 0 & 0 & \multirow{4}{*}{-0.9688} \\
\hline & $-1.24710 \mathrm{e}-15$ & 6.59346 & 0 & 0 & 0 & 0 & 0 & 0 & \\
\hline & $-2.35962 \mathrm{e}-7$ & 5.20107 & 0.042456 & 1 & 12.92183 & 0.0036148 & 1.03167 & 2.65582 & \\
\hline & -0.00052866 & 3.02216 & 0.025670 & 1 & 3.80507 & $9.27492 \mathrm{e}-5$ & 1 & 4.71131 & \\
\hline \multicolumn{10}{|c|}{ Влияние высоты над уровнем моря EEE8, м } \\
\hline \multirow{3}{*}{$\begin{array}{l}\text { EEE7 } \\
\text { Рис. } 3\end{array}$} & -87.70507 & 0 & $-8.09363 e-5$ & 1.07588 & 0 & 0 & 0 & 0 & \multirow{3}{*}{0.8611} \\
\hline & $2.50470 \mathrm{e}-6$ & 2.55776 & \begin{tabular}{|l|}
0.00026769 \\
\end{tabular} & 1.12617 & 0 & 0 & 0 & 0 & \\
\hline & $-9.59526 \mathrm{e}-8$ & 3.19687 & 0.0017158 & 0.99993 & 157.16690 & 0.28654 & 0.88567 & 6.01043 & \\
\hline \multirow{3}{*}{$\begin{array}{l}\text { EEE6 } \\
\text { Рис. } 4\end{array}$} & 7.25053 & 0 & -0.00032396 & 1.09546 & 0 & 0 & 0 & 0 & \multirow{3}{*}{0.8178} \\
\hline & $-6.85611 e-17$ & 5.03862 & 0 & 0 & 0 & 0 & 0 & 0 & \\
\hline & -0.00012515 & 2.24860 & 0.0020534 & 1 & 2922.5439 & -0.48413 & 0.99988 & -2.92162 & \\
\hline \multirow{3}{*}{$\begin{array}{l}\text { EЕE9 } \\
\text { Рис. } 5\end{array}$} & 59.61396 & 0 & -0.14457 & 0.46269 & 0 & 0 & 0 & 0 & \multirow{3}{*}{0.7201} \\
\hline & $-2.90947 \mathrm{e}-7$ & 3.11597 & 0 & 0 & 0 & 0 & 0 & 0 & \\
\hline & -0.00062264 & 2.34099 & 0.0018640 & 1.00057 & 846.23612 & -0.042840 & \begin{tabular}{|l|}
0.99814 \\
\end{tabular} & -0.80809 & \\
\hline \multicolumn{10}{|c|}{ Влияние годового баланса массы ледников ЕEЕ11, мм } \\
\hline \multirow{2}{*}{$\begin{array}{c}\text { EEE10 } 0^{3} \\
\text { Рис. } 6\end{array}$} & -28940.548 & 0 & 0 & 0 & 0 & 0 & 0 & 0 & \multirow{2}{*}{0.7592} \\
\hline & 270.01817 & 0.52442 & $2.61663 \mathrm{e}-5$ & 0.99761 & 0 & 0 & 0 & 0 & \\
\hline \multicolumn{10}{|c|}{ Влияние летнего баланса массы ледников, мм } \\
\hline \multirow{2}{*}{$\begin{array}{c}\text { EEE11 } \\
\text { Рис. } 7\end{array}$} & -11303.0999 & 0 & 0 & 0 & 0 & 0 & 0 & 0 & \multirow{2}{*}{0.7308} \\
\hline & 9.38697 & 0.76192 & 0 & 0 & 0 & 0 & 0 & 0 & \\
\hline
\end{tabular}

Примечания: ${ }^{1} x=x+90 ;{ }^{2} x=x+190 ;{ }^{3} x=x+12000$.

Из данных таблицы 5 видно, что для некоторых закономерностей потребовалось замена 
переменных из-за отрицательных значений у влияющих переменных.
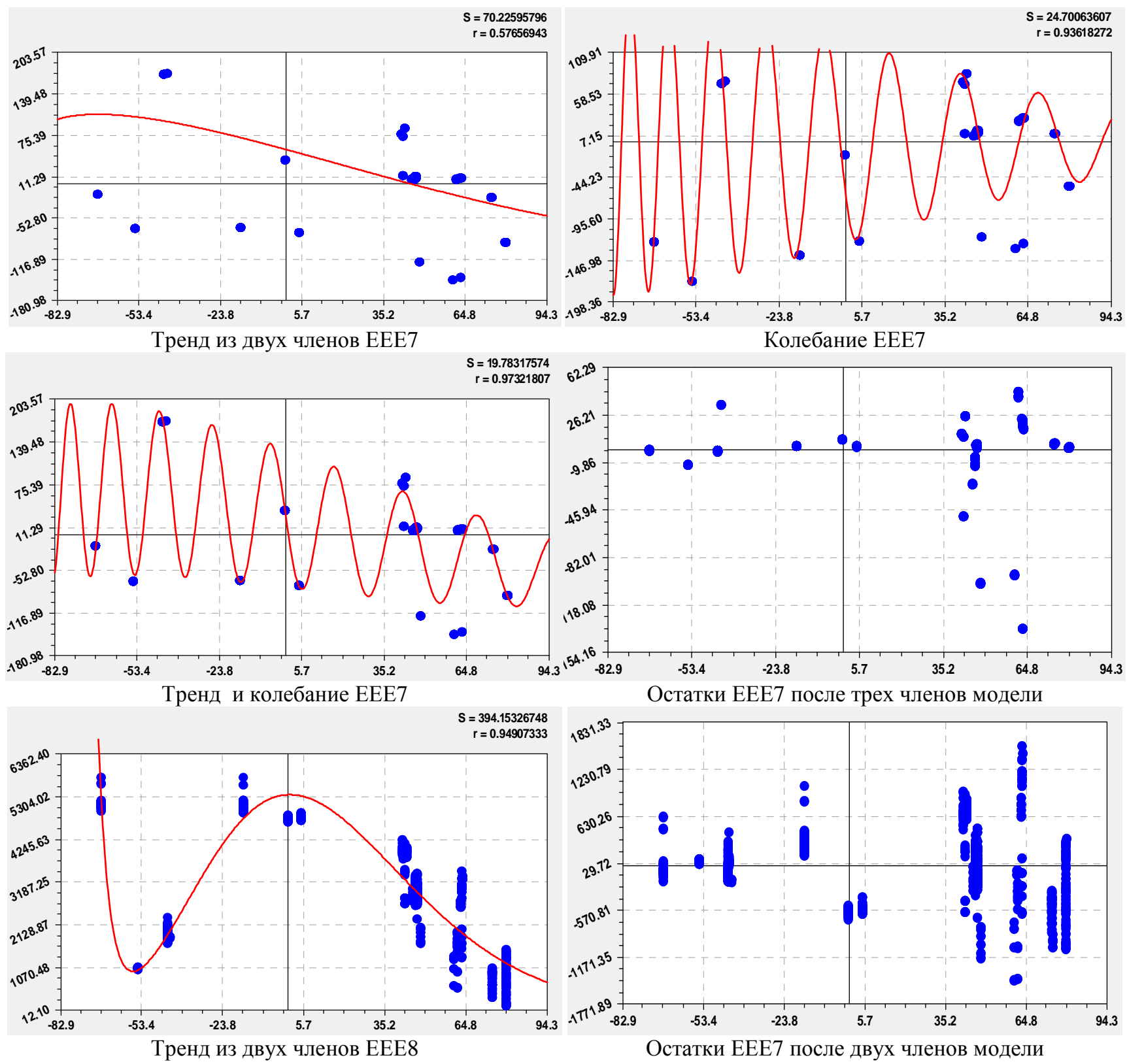

Рисунок 1. Графики моделей влияния фактора EEE6 на изменение факторов EEE7 и EEE8

Из графиков на рисунке 1 видно, что функция EEE7 = f(EEE6) содержит три члена, из которых первые два являются разностью двух законов: во-первых, закона экспоненциального роста; во-вторых, показательного закона. Третья составляющая является вейвлетом по формуле (2) с очень высоким коэффициентом корреляции 0.9362. Поэтому основной вклад в формирование бинарного отношения выполняет волновая функция с переменной амплитудой по закону экспоненциальной гибели и переменным периодом колебания. При этом начальный период колебания равен $2 \times 4.87479 \approx 9.75$ градусов. По мере увеличения широты колебание успокаивается из-за роста полупериода.

Остатки EEE7 после трех членов модели имеют значимые значения на широтах 40-65 градусов. Поэтому, если это необходимо, в дальнейшем возможен вейвлет-анализ микроволн по остаткам для этой широты.

Функция EEE8 = f(EEE6) состоит всего из двухчленного тренда. По данным таблицы 5 он содержит сумму из двух законов: во-первых, закона экспоненциальной гибели; во-вторых, био- 
технического закона [1-8]. Остатки показывают небольшое колебание, которое можно идентифицировать формулой (1), однако адекватность волновой составляющей будет очень малой.

Остальные графики имеют аналогичные объяснения. Поэтому ледники, как и другие виды природных объектов [1], имеют закономерные распределения.
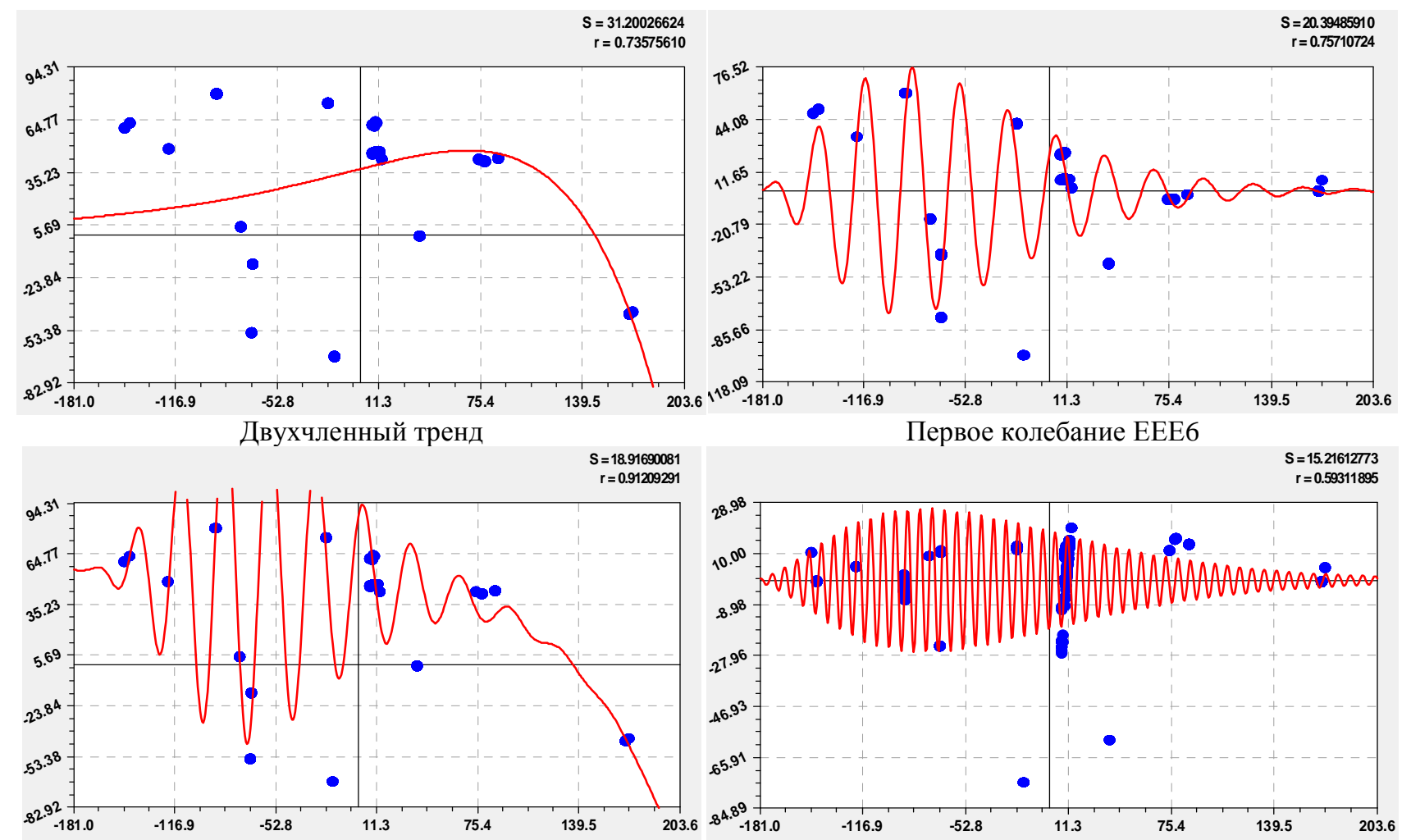

Тренд и колебание EEE6
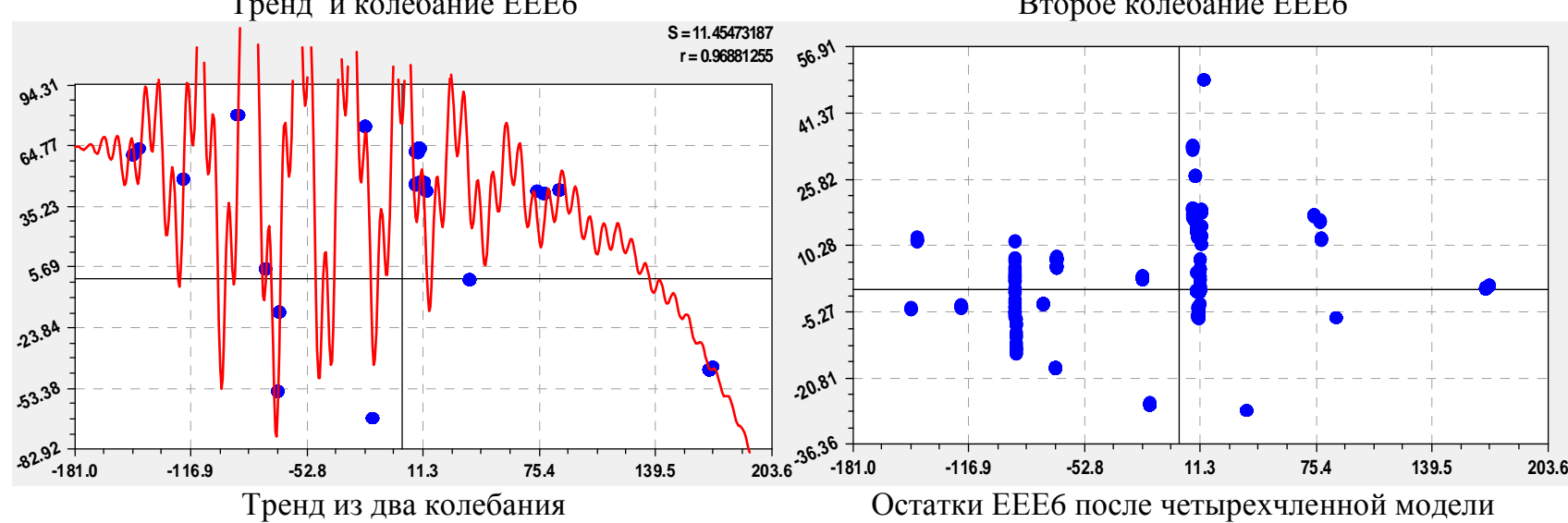

Рисунок 2. Графики моделей влияния EEE7 на изменение EEE6

Максимальное количество членов статистической модели EEE6 = f(EEE7) равно четырем, что соответствует вычислительным возможностям программной среды CurveExpert-1.40.

Для полного вейвлет-анализа необходимо разработать специальную программную среду по нашим сценариям статистического моделирования для суперкомпьютера петафлопного класса. При этом новая программная среда для больших объемов таблицы исходных данных будет универсальной для науки. 

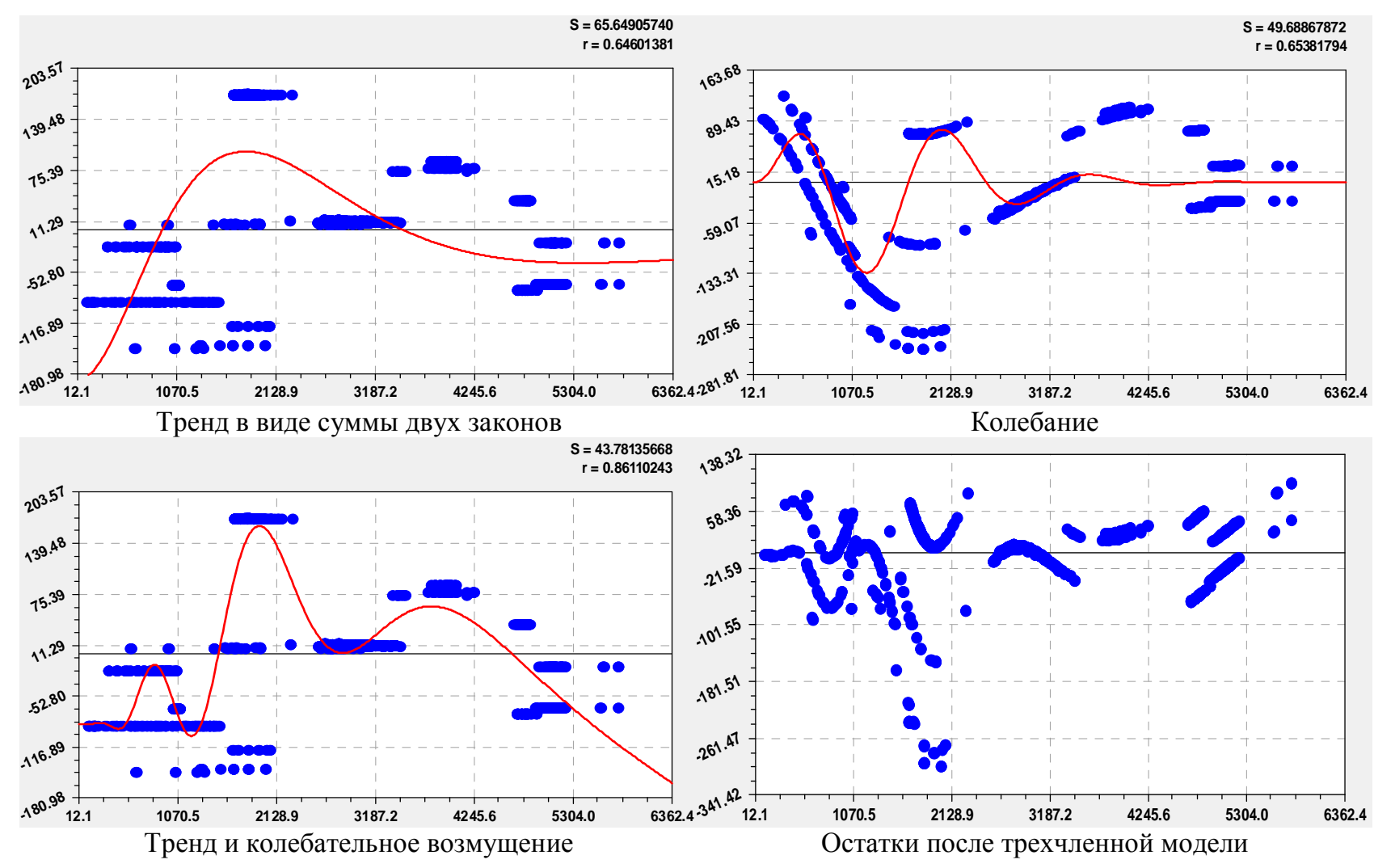

Рисунок 3. Графики моделей влияния EEE8 на изменение EEE7
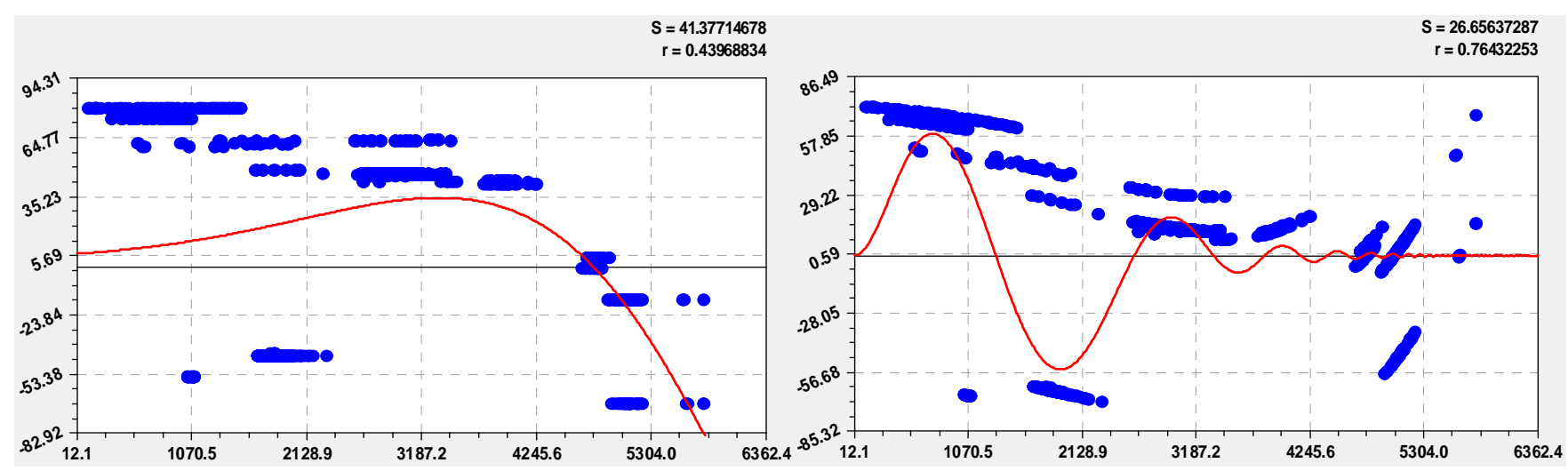
Тренд в виде суммы двух законов
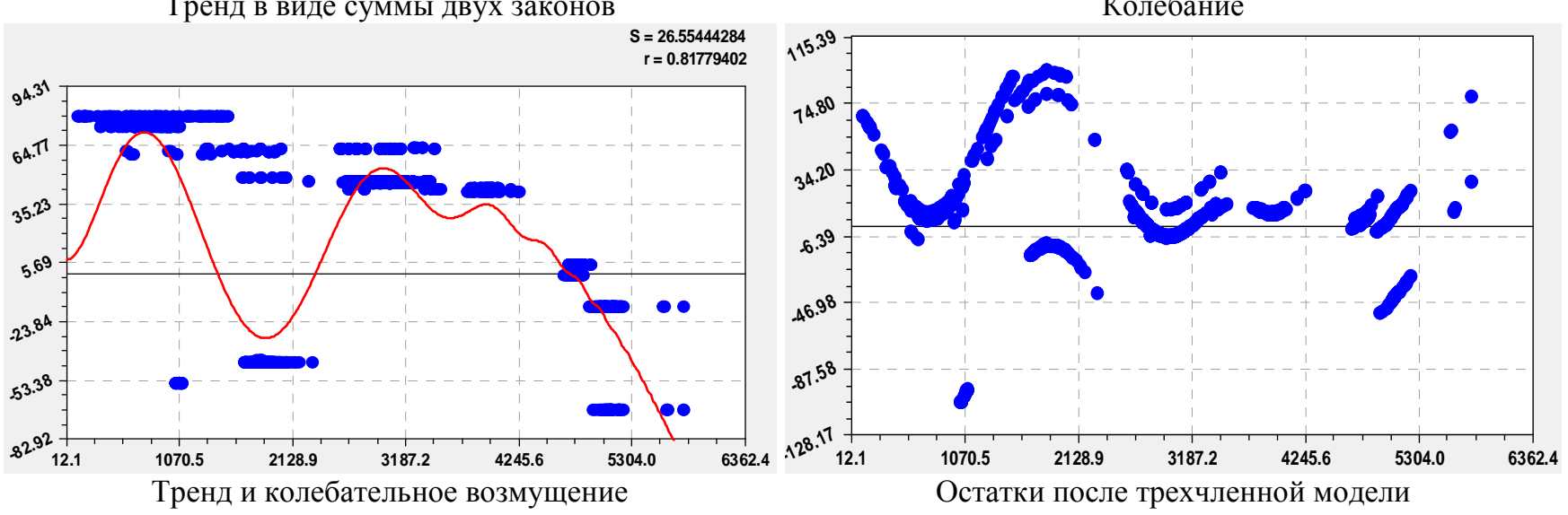

Рисунок 4. Графики моделей влияния EEE8 на изменение EEE6 

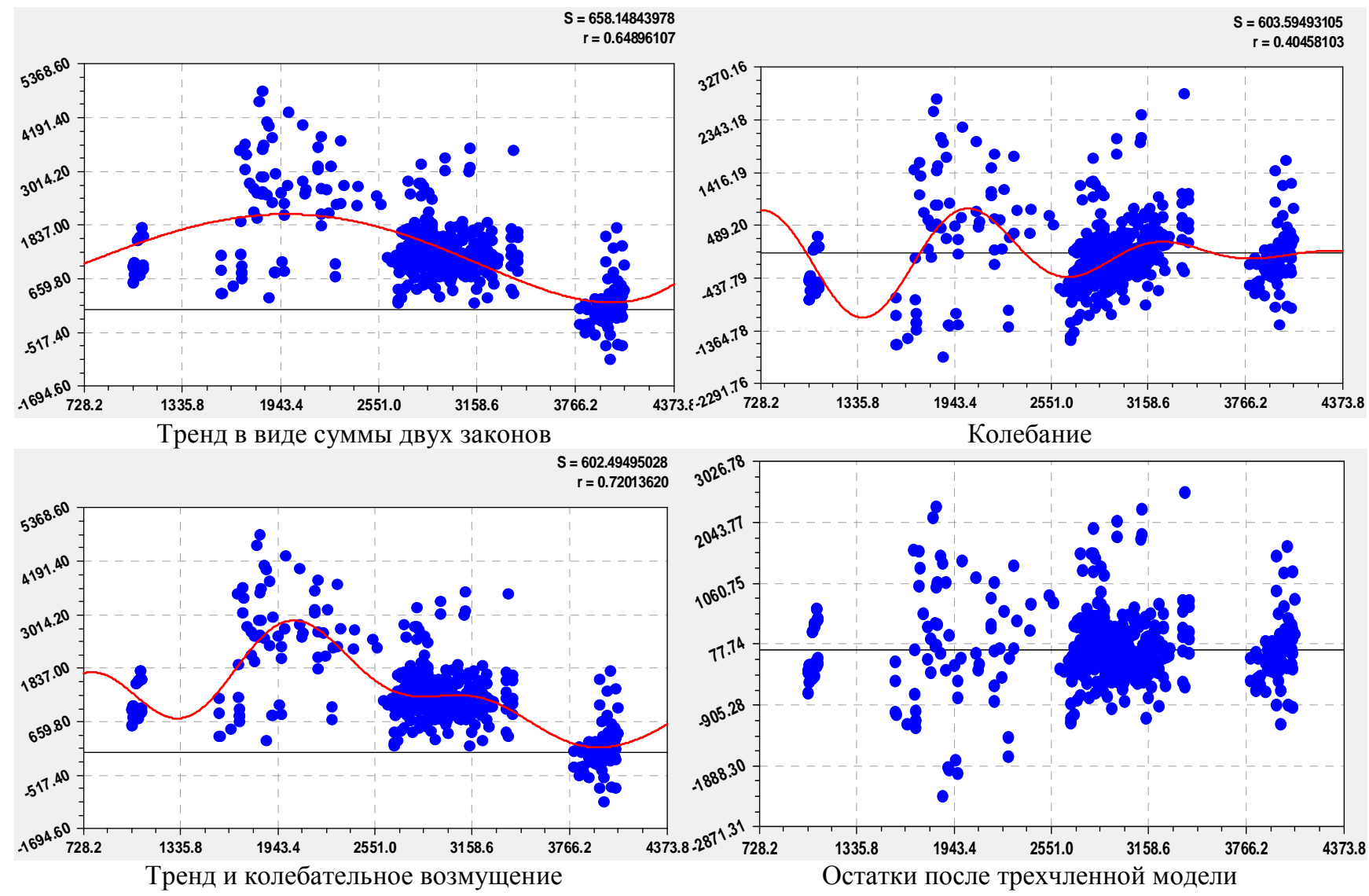

Рисунок 5. Графики моделей влияния EEE8 на изменение EEE9

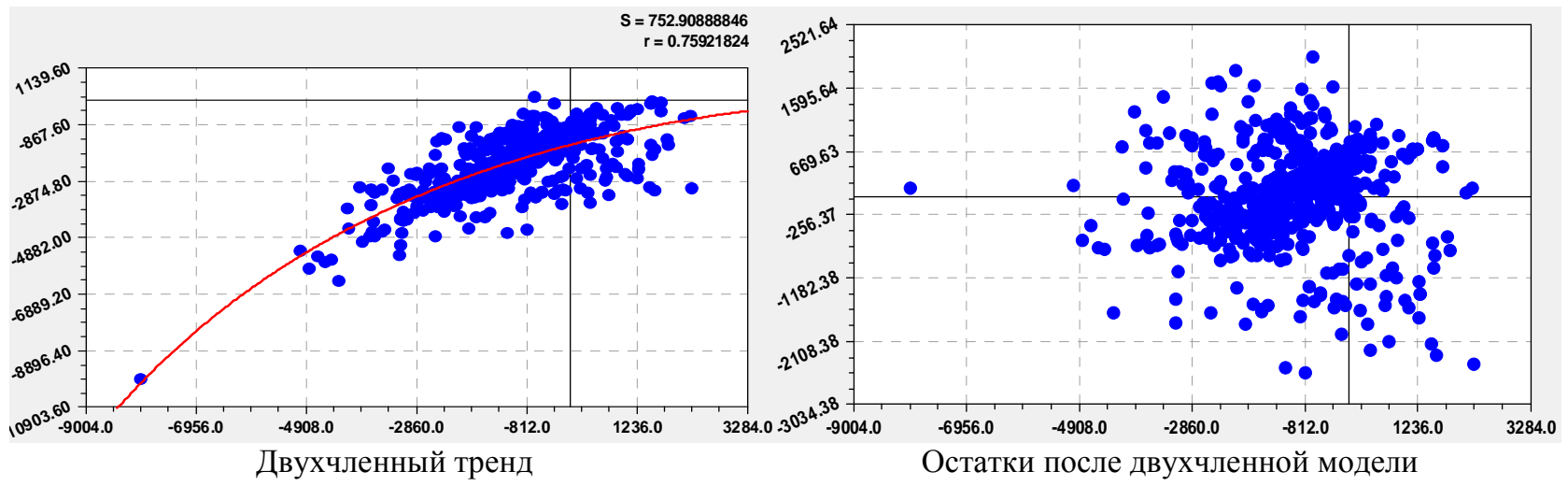

Рисунок 6. Графики моделей влияния EEE11 на изменение EEE10

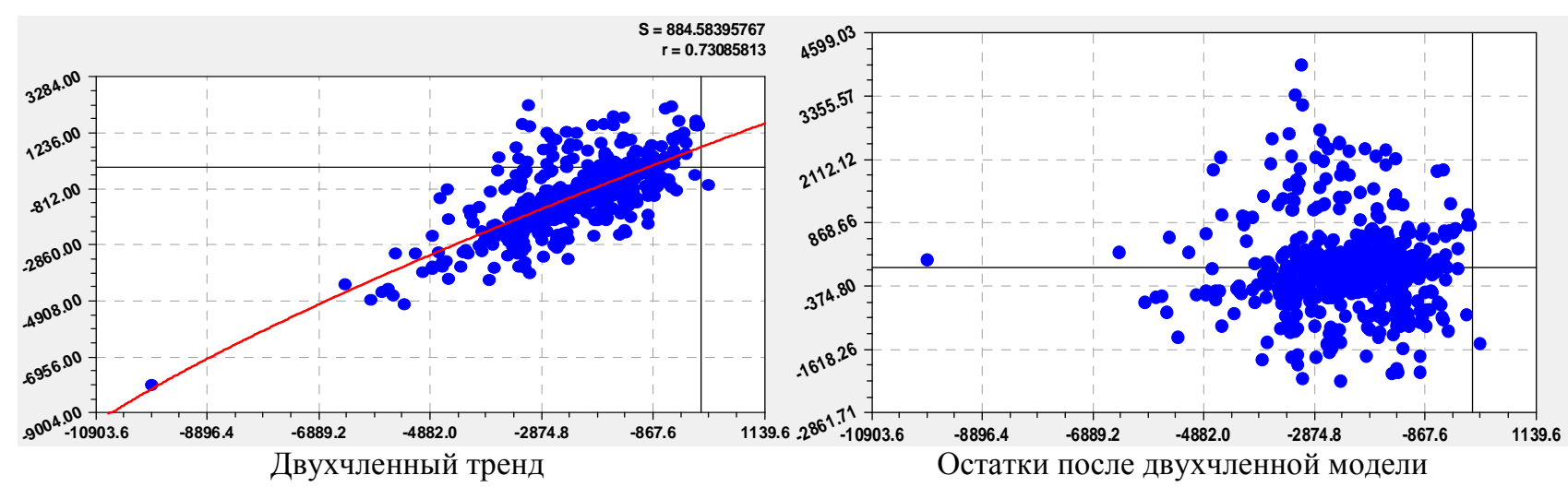

Рисунок 7. Графики моделей влияния EEE10 на изменение EEE11 
Анализ графиков по амплитудно-частотным характеристикам показывает, что система ледников обладает неким, пока неизвестным, свойством волновой адаптации к условиям существования на планете Земля.

Для некоторых бинарных отношений количество членов в общей статистической модели может превышать 100-120 шт. В этом случае появляется возможность проведения фрактального анализа для группировки вейвлетов по мега, макро, мезо и микроколебаниям.

\section{Выводы}

Доказана применимость статистической модели (1) к параметрам точек колебаний баланса у массы ледников Земли. В итоге каждое бинарное отношение содержит тренд и вейвлетсигналы. Причем тренд является частным случаем сверхдлинного по периоду колебания вейвлета. В итоге общая статистическая модель представляет собой жгут, состоящий из множества уединенных волн с переменными амплитудой и периодом колебаний. После статистического моделирования проводится факторный анализ, позволяющий составить рейтинги факторов как влияющих параметров и как зависимых показателей.

Предлагаемая методология идентификации позволяет выделить волны бинарных отношений между измеренными факторами у ледников. При этом для 2528 ледников подгруппы ЕЕЕ характерны конечномерные вейвлеты, которые в дальнейшем можно сопоставлять с эвристическими представлениями специалистов. Метод идентификации позволяет выделить значимые параметры ледников и бинарные отношения между ними, по которым нужно будет повышать точность будущих измерений. При этом из разных подгрупп нами были выделены 26 факторов, но их совместному анализу мешает нестыковка между таблицами данных.

\section{Литература}

1. П.М. Мазуркин. Геоэкология: Закономерности современного естествознания: научное изд. Йошкар-Ола: МарГТУ, 2006. 336 с.

2. П.М. Мазуркин. Идентификация статистических устойчивых закономерностей // Наука и мир: международный научный журнал. 2013. № 3(3). С.28-33.

3. П.М. Мазуркин. Коррелятивная вариация: учеб. пос. с грифом УМО РАЕ. Йошкар-Ола: Поволжский ГТУ, 2013. 120 с.

4. П.М. Мазуркин. Лесоаграрная Россия и мировая динамика лесопользования. ЙошкарОла: МарГТУ, 2007. 334 с.

5. П.М. Мазуркин. Решение 23-ой проблемы Гильберта Междисциплинарные исследования в области математического моделирования и информатики. Матер. 3-й научно-прак. internet-конф. Ульяновск: SIMJET, 2014. С 269-277.

6. П.М. Мазуркин. Статистическое моделирование. Эвристико-математический подход. Йошкар-Ола: МарГТУ, 2001. 100с.

7. П.М. Мазуркин, С.И. Михайлова. Территориальное экологическое равновесие = Territprial ecological balance: аналит. обзор; Учреждение Рос. акад. наук Гос. публич. науч.-техн. б-ка Сиб. отд-ния РАН, М-во образования и науки Рос. Федерации Федер. Марийс. гос. техн. ун-т. Новосибирск : ГПНТБ СО РАН, 2010. 430 с. (Сер. Экология. Вып. 94).

8. П.М. Мазуркин, А.С. Филонов. Математическое моделирование. Идентификация однофакторных статистических закономерностей: учеб. пос. Йошкар-Ола: МарГТУ, 2006. 292 с.

9. Fluctuations of Glaciers Database. World Glacier Monitoring Service (WGMS). 2014. FoGDB-Version_2013-11. http://wgms.ch/data-exploration/database-versions/. DOJ 10.5904/wgms-fog2013-11.

10. P.M. Mazurkin. Invariants of the Hilbert Transform for 23-Hilbert Problem, Advances in Sciences and Humanities. Vol. 1, No. 1, 2015, pp. 1-12. doi: 10.11648/j.ash.20150101.11

11. P.M. Mazurkin. Method of identification // $14^{\text {th }}$ International multidisciplinary scientific geoconferenct \& SGEM2014. GeoConference jn NANO. BIO AND GREEN - TECHNOLOGIES FOR A SUSTAINABLE FUTURE. Conference proceedincs. Volume 1. Section Advances in Biotech- 
nology. 17-26 June 2014. Albena. Bulgaria. P. 427-434.

12. P.M. Mazurkin. Statistical modeling of entire prime numbers / International Journal of Engineering and Technical Research (IJETR) ISSN: 2321-0869. Volume-2. Issue-8. August 2014. P.148158.

\title{
IDENTIFICATION OF TRENDS AND WAVE PATTERNS ACCORDING TO THE STATISTICS OF FLUCTUATIONS IN GLACIER MASS
}

\author{
P.M. Mazurkin \\ Doctor of Engineering, professor, Academician of Russian Academy of Natural History \\ and Russian Academy of Natural Sciences, member of the European Academy of \\ Natural sciences, Volga State University of Technology, Yoshkar-Ola, Russia, kaf_po@mail.ru
}

\begin{abstract}
Identification method shown in the example of table data of measurements of the six parameters of the subgroup EEE on oscillations in 2013 mass balance 2528 glaciers of the Earth. The equations of the trend and oscillatory perturbations on the basis of stable laws for a generalized wave function in the form of asymmetric wavelet signal with variable amplitude and period of oscillation. Graphics components of the generalized model of the wavelet waveform, you can visually see a picture of the mutual influence of all six parameters of the subgroup EEE fluctuations in glacier mass. On identified equations can spend the amplitude-frequency analysis.
\end{abstract}

Keywords: glaciers, oscillation, mass balance, factors, patterns

\section{References}

[1] Fluctuations of Glaciers Database. World Glacier Monitoring Service (WGMS). 2014. FoG-DBVersion_2013-11. http://wgms.ch/data-exploration/database-versions/. DOJ 10.5904/wgms-fog2013-11.

[2] P.M. Mazurkin. Biotechnical law and designing of adequate models // Achievements of modern natural sciences 2009. No. 9. P. 125-129.

[3] P.M. Mazurkin. The biotechnical law, algorithm in intuitive sense and algorithm of search of parameters // Achievements of modern natural sciences. 2009. №. 9. P. 88-92.

[4] P.M. Mazurkin. The biotechnical principle in statistical modeling // Achievements of modern natural sciences. 2009 . No. 9. P. 107-111.

[5] P.M. Mazurkin. Biotechnical principle and steady laws of distribution // Achievements of modern natural sciences. 2009. No. 9 of Page 93-97. URL: www.rae.ru/use/?section=content\&op= show article\&article_id=7784060.

[6] P.M. Mazurkin. Geoecology: Patterns of Modern Natural Science. Yoshkar-Ola: MarSTU, 2006. $336 \mathrm{p}$.

[7] P.M. Mazurkin. Identification of statistical steady regularities // Science and world: international scientific magazine. 2013. № 3(3). P. 28-33.

[8] P.M. Mazurkin. Invariants of the Hilbert Transform for 23-Hilbert Problem, Advances in Sciences and Humanities. Vol. 1, No. 1, 2015, pp. 1-12. doi: 10.11648/j.ash.20150101.11

[9] P.M. Mazurkin. Method of identification // $14^{\text {th }}$ International multidisciplinary scientific geoconferenct \& SGEM2014. GeoConference jn NANO. BIO AND GREEN - TECHNOLOGIES FOR A SUSTAINABLE FUTURE. Conference proceedincs. Volume 1. Section Advances in Biotechnology. 17-26 June 2014. Albena. Bulgaria. P. 427-434.

[10] P.M. Mazurkin. The decision 23-oh Gilbert's problems. Interdisciplinary researches in the field of mathematical modeling and informatics. Materials of the 3rd scientific and practical Internet conference. Ulyanovsk: SIMJET. 2014. P. 269-277.

[11] P.M. Mazurkin. Statistical modeling of entire prime numbers / International Journal of Engineering and Technical Research (IJETR) ISSN: 2321-0869. Volume-2. Issue-8. August 2014. P.148158 . 
[12] P.M. Mazurkin. A.S. Filonov. Mathematical modeling. Identification of one-factorial statistical regularities: manual. Yoshkar-Ola: MarSTU. 2006. 292 p. 